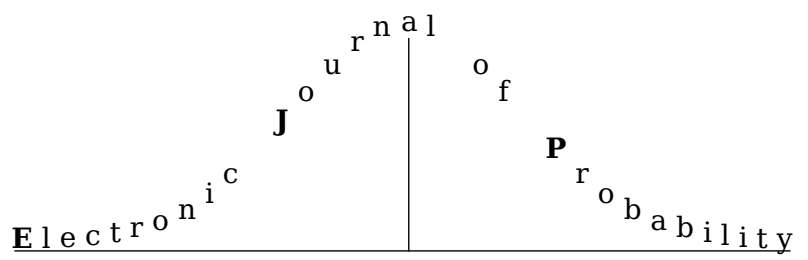

Electron. J. Probab. 25 (2020), article no. 107, 1-15.

ISSN: 1083-6489 https://doi.org/10.1214/20-EJP508

\title{
Necessary and sufficient conditions for the finiteness of the second moment of the measure of level sets
}

\author{
Jean-Marc Azaïs* José R. León ${ }^{\dagger}$
}

\begin{abstract}
For a smooth vectorial stationary Gaussian random field, $X: \Omega \times \mathbb{R}^{d} \rightarrow \mathbb{R}^{d}$, we provided necessary conditions to have a finite second moment for the number of roots of $X(t)-u$. Then, under a more restrictive hypothesis, some sufficient conditions were also given. The results were obtained using a method of proof inspired the one obtained by D. Geman for stationary Gaussian processes. Afterward, the same method is applied to the number of critical points of a scalar random field and to the level set of a vectorial process, $X: \Omega \times \mathbb{R}^{D} \rightarrow \mathbb{R}^{d}$, with $D>d$.
\end{abstract}

Keywords: level sets; Kac-Rice formula; moments; random fields.

MSC2020 subject classifications: $60 \mathrm{G} 15 ; 60 \mathrm{G} 60$.

Submitted to EJP on June 17, 2019, final version accepted on August 10, 2020.

\section{Introduction}

In the 1940s, three articles with apparently different orientations appeared in the mathematical literature. The first was Mark Kac's paper [12], “On the average number of real roots of a random algebraic equation", and the other two were two papers written by S.O. Rice [18, 19], "Mathematical analysis of random noise I and II". In the work of Kac and in the second paper of Rice, the zeros of Gaussian random functions were studied. In particular, they established with precision a formula, known today as the Kac-Rice formula, which allows computing the expectation of the number of zeros (or crossings by any level) of a Gaussian random function. Although the works seem rather dissimilar, M. Kac in the review of the article affirms that "All these results (of Rice) can also be derived using the methods introduced by the reviewer (Kac)".

An intense research activity has been developed after these two works. In particular, interest in these topics grew considerably after the publication of the book written by $\mathrm{H}$. Cramer and M. R. Leadbetter [8]. In this work, there is not only a general demonstration of the Kac-Rice formula for the number of crossings of Gaussian processes but also

\footnotetext{
*IMT, Université de Toulouse, Toulouse, France. E-mail: jean-marc.azais@math. univ-toulouse. fr

${ }^{\dagger}$ IMERL, Facultad de Inegeniería Universidad de la República, Montevideo, Uruguay and Universidad Central de Venezuela. E-mail: rlramos@fing.edu.uy
} 
formulas for their factorial moments. An important fact is that in the book, a sufficient condition for the second moment of the number of crossings of zero to be finite is established. Later D. Geman in [11] showed that this condition was also necessary. Since then this necessary and sufficient condition is called "Geman Condition". This result has been extended to any level in [13].

The theme gained a new impulse in the eighties when two books appear. The first one written by R. Adler [1], "The geometry of random fields" and the second one [20] by $\mathrm{M}$. Wschebor "Surfaces aléatoires. Mesure géométrique des ensembles de niveau". Both books focus on crossings or geometric invariants of the level sets for random fields having a multidimensional domain and taking scalar or vector values. The problems studied by Cramer \& Leadbetter were extended to this new context. In particular, we must point out Adler \& Hasofer's article [2] in which conditions are established so that the number of stationary points for a Gaussian field, $X: \mathbb{R}^{2} \rightarrow \mathbb{R}$, have a second moment. It is important to observe that studying the stationary points of a scalar field leads to study the zeros of its gradient, which is a vector field.

The twenty-first century saw two books appear, [3] and [6], that gave a new impetus to the subject. New fields of application of such formulas appeared in the literature, and the area has become a large domain of research. We can point out, for instance, the applications to the number of roots of random polynomial systems (algebraic or trigonometric) and to the volume of nodal sets when the systems are rectangular [17]. Also, Kac-Rice formulas are basic tools, to study the sets of zeros of random waves and many efforts have been made to prove or disprove Berry's conjectures [7] (see [17] and the references therein). A field of applications where these formulas have been very useful is random sea modeling. The Lund's School of probability has been very active in these matters (see for instance the paper [16] and the references therein). Also, the processes, which the crossings are studied can have their domain in a manifold of finite dimension (see [15]). A very interesting case of this last situation is the article [5], where the domain of the random field is the sphere in large dimension.

In the present paper, we obtain necessary and sufficient conditions to have a finite second moment for the number of roots of $X(t)-u$, for a stationary, mean zero Gaussian field, $X: \Omega \times \mathbb{R}^{d} \rightarrow \mathbb{R}^{d}$. The proofs of the main results are rather simple using the case $d=1$ as inspiration. Our results can be extended to the number of critical points of a stationary mean zero scalar Gaussian field. We must note that in [9] was given a sufficient condition for the critical points of a scalar field to have finite second moment. However, our method is different. To be more precise, their method is based on the study of the conditional expectation that emerges in the Kac-Rice formula in a neighborhood of zero. Then they link this expression to the behavior of the fourth derivative of the covariance function. Instead, our proof uses geometric arguments. Finally, let us point out that as a bonus, our method of proof allows obtaining a very simple result for the volume of level sets for Gaussian fields, $X: \Omega \times \mathbb{R}^{D} \rightarrow \mathbb{R}^{d}$, with $D>d$. Under conditions of stationarity and differentiability, the second moment is always finite.

Suppose we have an easy way to check the measure of the level set of a Gaussian field has a finite second moment. Then, one can readily obtain an Itô-Wiener expansion for this functional. Two consequences of this representation are important to remark: first, the exact asymptotic variance of the level functional can be computed second, the fourth-moment theorem can be used to obtain diverse CLT. This has been done in [14] and more recently in a lot of papers. We can cite the article [17], where one can also consult some recent references.

The paper es organized as follows. In Section 2, we revisit the results of [13] in dimension 1. Section 3 studies the number of points of levels sets for a random field, $X: \mathbb{R}^{d} \rightarrow \mathbb{R}^{d}, d>1$. The subsection 3.4 is devoted to the study of the number of critical 
points of a random field, $X: \mathbb{R}^{d} \rightarrow \mathbb{R}$. Section 4 studies the measure of levels sets for a random field, $X: \mathbb{R}^{D} \rightarrow \mathbb{R}^{d}, D>d$. The proofs of the different lemmas are given in the appendix.

\section{Real valued process on the line, Geman's condition}

The results of this section are in the paper [13]. However, we present a new proof as an introduction to the next section.

Consider a process $X: \mathbb{R} \rightarrow \mathbb{R}$ and assume,

- It is Gaussian, stationary, and normalized to have:

$$
\mathbb{E}(X(0))=0 ; \quad \operatorname{Var}(X(t))=1 .
$$

Last point is without loss of generality.

- The second spectral moment $\lambda_{2}$ is positive and finite. The finiteness of this quantity implies that $X$ is differentiable in quadratic mean. Denoting $X^{\prime}$ its derivative. Moreover, it holds $\lambda_{2}=-r^{\prime \prime}(0)=\mathbb{E}\left[\left(X^{\prime}(0)\right)^{2}\right]$.

Let $N_{u}([0, T]):=\#\{t \in[0, T]: X(t)=u\}$ for a given level $u \in \mathbb{R}$. Moreover, we define the covariance

$$
r(\tau)=\mathbb{E}[X(0) X(\tau)]
$$

Set

$$
\sigma^{2}(\tau):=\operatorname{Var}\left(X^{\prime}(0) \mid X(0)=X(\tau)=0\right)=\lambda_{2}-\frac{\left(r^{\prime}(\tau)\right)^{2}}{1-r^{2}(\tau)} .
$$

In what follows, (Const) will denote a generic positive constant, its value can change from one occurrence to another. The relation $x \leq($ Const $) y, y \leq($ Const $) x$ is denoted $x \asymp y$.

The object of this section is to prove the following theorem:

Theorem 2.1. The following statements are equivalent

(a) $\mathbb{E}\left(N_{u}([0, T])^{2}\right)$ is finite for some $u$ and $T$.

(b) $\mathbb{E}\left(N_{u}([0, T])^{2}\right)$ is finite for all $u$ and all finite $T$.

(c) The integral $\int \frac{\sigma^{2}(\tau)}{\tau} d \tau$ converges at zero.

Remark Integrating by parts in (c), we get the classical Geman's condition by using the following lemma, whose proof is found in the appendix.

Lemma 2.2. There is an equivalence between the convergence at zero of the two following integrals

$$
\int \frac{r^{\prime \prime}(\tau)-r^{\prime \prime}(0)}{\tau} d \tau=\int \frac{\lambda_{2}+r^{\prime \prime}(\tau)}{\tau} d \tau \quad \text { and } \quad \int \frac{\sigma^{2}(\tau)}{\tau} d \tau .
$$

Before, the proof of the theorem, we need some notation and two lemmas.

Lemma 2.3. For $\tau$ sufficiently small, we set the following definitions and we have the following relations.

(a) $\mu_{1, \tau, u}:=\mathbb{E}\left(X^{\prime}(\tau) \mid X(0)=X(\tau)=u\right)=\frac{r^{\prime}(\tau) u}{1+r(\tau)}$.

(b) $\mu_{2, \tau, u}:=\mathbb{E}\left(X^{\prime}(0) \mid X(0)=X(\tau)=u\right)=-\mu_{1, \tau, u}$. 
(c) Recall that $\sigma^{2}(\tau)=\operatorname{Var}\left(X^{\prime}(0) \mid X(0), X(\tau)\right)=\lambda_{2}-\frac{\left(r^{\prime}(\tau)\right)^{2}}{1-r^{2}(\tau)}$.

(d) $\operatorname{det}\left(\operatorname{Cov}(X(0) ; X(\tau))=1-r^{2}(\tau) \asymp \tau^{2}\right.$.

(e) If the fourth spectral moment $\lambda_{4}$ satisfies $\lambda_{2}^{2}<\lambda_{4} \leq+\infty$, then $\frac{\left|\mu_{1, \tau, u}\right|}{\sigma(\tau)} \leq($ Const $) u$.

Lemma 2.4. Assume that $\left|m_{1}\right|,\left|m_{2}\right| \leq K$ for some constant $K$ and that $\left(Y_{1} ; Y_{2}\right) \stackrel{d}{=}$ $N\left(\left(m_{1} ; m_{2}\right),\left(\begin{array}{ll}1 & \rho \\ \rho & 1\end{array}\right)\right)$. Then, $\mathbb{E}\left|Y_{1} Y_{2}\right| \asymp 1$. The two constants implied in the symbol depend on $K$.

Proof of the Theorem. First we have to consider the particular case $\lambda_{4}=\lambda_{2}^{2}$. This corresponds to the Sine-Cosine process: $X(t)=\xi_{1} \sin (w t)+\xi_{2} \cos (w t)$, where $\xi_{1}, \xi_{2}$ are independent standard Gaussians. In this case, a direct calculation shows that (a)-(c) hold true.

We now consider the other cases assuming that $\lambda_{2}^{2}<\lambda_{4}$. We start from (c), assuming that

$$
\int_{0}^{T} \frac{\sigma^{2}(\tau)}{\tau} d \tau<+\infty \quad \text { with } T \text { sufficiently small. }
$$

The expectation of the number of crossings is finite because the second spectral moment is (see [8]). Thus, it is enough to work with the second factorial moment. Since $0<\lambda_{2}<+\infty$ and since at zero $r(\tau)=1+\lambda_{2} \tau^{2}+o\left(\tau^{2}\right),|r(\tau)| \neq 1$ for $\tau$ sufficiently small (which has been assumed). This implies that the Kac-Rice formula for this quantity [8] holds true and writes

$$
\begin{aligned}
& \mathbb{E}\left(N_{u}([0, T])\left(N_{u}([0, T])-1\right)\right)= \\
& \quad \frac{1}{\pi} \int_{0}^{T}(T-\tau) \mathbb{E}\left(\left|X^{\prime}(0)\right|\left|X^{\prime}(\tau)\right| \mid X(0)=X(\tau)=u\right) \frac{e^{-\frac{u^{2}}{1+r}}}{\sqrt{1-r^{2}}} d \tau \\
& \quad \leq T(\text { Const }) \int_{0}^{T} \mathbb{E}\left(\left|\frac{X^{\prime}(0)}{\sigma(\tau)} \| \frac{X^{\prime}(\tau)}{\sigma(\tau)}\right| \mid X(0)=X(\tau)=u\right) \frac{\sigma^{2}(\tau)}{\tau} d \tau
\end{aligned}
$$

using Lemma $2.3(\mathrm{~d})$. The random variables $\frac{X^{\prime}(0)}{\sigma(\tau)}$ and $\frac{X^{\prime}(\tau)}{\sigma(\tau)}$ have a bounded conditional mean by Lemma 2.3 (e), applying now Lemma 2.4:

$$
\mathbb{E}\left(N_{u}([0, T])\left(N_{u}([0, T])-1\right) \leq T(\text { Const }) \int_{0}^{T} \frac{\sigma^{2}(\tau)}{\tau} d \tau .\right.
$$

Hence, the finiteness of the second moment holds, for $T$ sufficiently small. Moreover, Minkowski inequality proves that it is also the case for every $T$, this proves (b).

In the other direction, we start from (a), with $u=0$ and $T$ sufficiently small (which is weaker than (b)) and we prove (c).

Again, we can consider the second factorial moment and apply the Kac-Rice formula to get

$$
\begin{aligned}
& \mathbb{E}\left(N_{u}([0, T])\left(N_{u}([0, T])-1\right)\right. \\
& \quad \geq(\text { Const }) \int_{0}^{T / 2} \mathbb{E}\left(\left|\frac{X^{\prime}(0)}{\sigma(\tau)}\right|\left|\frac{X^{\prime}(\tau)}{\sigma(\tau)}\right| \mid X(0)=X(\tau)=u\right) \frac{\sigma^{2}(\tau)}{\tau} d \tau .
\end{aligned}
$$

It suffices to apply Lemma 2.4 in the other direction.

Remark 2.5. We can also obtain (2.2) with an explicit constant by use of the CauchySchwarz inequality. 


\section{Random fields $\mathbb{R}^{d}$ to $\mathbb{R}^{d}, d>1$}

\subsection{Position of the problem}

Let us consider a random field, $X: \mathbb{R}^{d} \rightarrow \mathbb{R}^{d}$. We assume $\left(H_{1}\right)$ :

- The field is Gaussian and stationary and has a continuous derivative.

- The distribution of $X(0)$ (respectively $\left.X^{\prime}(0)\right)$ is non degenerate (N.D.).

By a rescaling in space, we can assume without loss of generality that

$$
\mathbb{E}[X(t)]=0 \quad \text { and } \quad \operatorname{Var}(X(t))=I_{d}
$$

where Var denotes the variance-covariance matrix. We keep the notation Cov for the matrix

$$
\operatorname{Cov}(X, Y):=\mathbb{E}\left((X-\mathbb{E}(X))(Y-\mathbb{E}(Y))^{\top}\right)
$$

We also define the following additional hypothesis.

We define

$$
\begin{aligned}
\sigma_{i, \lambda}^{2}(r) & :=\operatorname{Var}\left(X_{i \lambda}^{\prime} \mid X(0), X(\lambda r)\right), \\
\sigma_{\max }^{2}(r) & :=\max _{i=1, \ldots, d} \max _{\lambda \in \mathbb{S}^{d-1}} \sigma_{i, \lambda}^{2}(r),
\end{aligned}
$$

where $X_{i \lambda}^{\prime}$ denotes the derivative of $X_{i}$ in the direction $\lambda \in \mathbb{S}^{d-1}$.

\subsection{Zero level}

In this section, we limit our attention to the number of zeros $N(0, S)$ of the random field $X$ on some compact set $S$. The following result is new as the rest of the section.

Theorem 3.1. Under $(H 1)$, if

$$
\int \frac{\sigma_{\max }^{2}(r)}{r} d r \text { converges at } 0,
$$

then for all compact $S \subset \mathbb{R}^{d}: \mathbb{E}\left((N(0, S))^{2}\right)$ is finite.

Remark 3.2. We conjeture that the result is true for all levels and that

$$
\int \frac{\sigma_{\min }^{2}}{r}(r)<\infty
$$

is a necessary condition, where $\sigma_{\min }^{2}(r):=\min _{i=1, \ldots, d} \max _{\lambda \in \mathbb{S}^{d-1}} \sigma_{i, \lambda}^{2}(r)$, but the computations are not tractable.

The proof of the theorem uses the following lemma.

Lemma 3.3. Let $T,\left(Z_{n}\right)_{n}$ be in the same Gaussian space. Assume that $Z_{n} \rightarrow Z$ a.s. or in probability or in $\mathbb{L}^{2}(\Omega)$ and the random variable $Z$ is (N.D.). Then

$$
\begin{gathered}
\forall z, \mathbb{E}\left(T \mid Z_{n}=z\right) \rightarrow \mathbb{E}(T \mid Z=z), \\
\operatorname{Var}\left(T \mid Z_{n}\right) \rightarrow \operatorname{Var}(T \mid Z) .
\end{gathered}
$$


Proof of Theorem 3.1. Set

$$
\mathcal{C}=\{X(0)=X(t)=u\}
$$

and let $E_{\mathcal{C}}$ denote the expectation conditional to $\mathcal{C}$.

We consider the following quantity

$$
\mathcal{A}(t, u)=\mathbb{E}_{\mathcal{C}}\left(\left|\operatorname{det} X^{\prime}(0) \operatorname{det} X^{\prime}(t)\right|\right) .
$$

By applying the Cauchy-Schwarz inequality and symmetry of the roles of 0 and $t$ :

$$
\mathcal{A}(t, u) \leq \mathbb{E}_{\mathcal{C}}\left(\operatorname{det}\left(\left(X^{\prime}(0)\right)^{\top} X^{\prime}(0)\right)\right),
$$

We define the Jacobian of the matrix $X^{\prime}(0)$ by $X_{i j}^{\prime}=\frac{\partial X_{i}}{\partial t_{j}}$.

We remark that, again by triangular inequality, it is sufficient to prove the finiteness of $\mathbb{E}\left((N(0, S))^{2}\right)$ for $S$ sufficiently small.

We perform a change of basis so that $t=r e_{1}=|t| e_{1}$, where $e_{1}$ is the first vector of the new basis. We denote by $\bar{X}$ the expression of $X$ in this basis. Let $\bar{X}_{: j}^{\prime}$ denote the $j$ th column of $\bar{X}^{\prime}$. Using Hadamard's inequality for the matrix $M=\left(M_{i j}\right)$, we know that

$$
\operatorname{det}(M) \leq M_{1,1} \ldots M_{d, d}
$$

This gives

$$
\mathcal{A}(t, u) \leq \mathbb{E}_{\mathcal{C}}\left(\left\|\bar{X}_{: 1}^{\prime}\right\|^{2} \ldots\left\|\bar{X}_{: d}^{\prime}\right\|^{2}\right)=\sum_{1 \leq i_{1}, \ldots, i_{d} \leq d} \mathbb{E}_{\mathcal{C}}\left(\left(\bar{X}_{i_{1}, 1}^{\prime}\right)^{2} \ldots\left(\bar{X}_{i_{d}, d}^{\prime}\right)^{2}\right)
$$

Because the conditional expectation is contractive, for $j>1$,

$$
\mathbb{E}_{\mathcal{C}}\left(\left(\bar{X}_{i_{j}, j}^{\prime}\right)^{2}\right) \leq \mathbb{E}\left(\left(\bar{X}_{i_{j}, j}^{\prime}\right)^{2}\right) \leq(\text { Const }) .
$$

In addition

$$
\mathbb{E}_{\mathcal{C}}\left(\left(\bar{X}_{i_{1}, 1}^{\prime}\right)^{2}\right) \leq \sigma_{\max }^{2}(r) .
$$

If we consider a term of (3.3), we can apply the Cauchy-Schwarz inequality to get that it is bounded by

$$
\left(\mathbb{E}_{\mathcal{C}}\left(\left(\bar{X}_{i_{1}, 1}^{\prime}\right)^{4}\right)^{1 / 2}\left(\mathbb{E}_{\mathcal{C}}\left(\left(\bar{X}_{i_{2}, 2}^{\prime}\right)^{4} \ldots\left(\bar{X}_{i_{d}, d}^{\prime}\right)^{4}\right)\right)^{1 / 2}\right.
$$

Using (3.4) and (3.5), we get that this term is bounded by

$$
\text { (Const) } \sigma_{\max }^{2}(r) \text {. }
$$

As a consequence, we get the same bound for the whole sum.

We now study the joint density

$$
p_{X(0), X(t)}(0,0)=(\text { Const })(\operatorname{det} \operatorname{Var}(X(0), X(t)))^{-\frac{1}{2}} .
$$

Using the fact that a determinant is invariant by adding to some row (or column) a linear combination of the others rows (or columns) we get

$$
\operatorname{det}(\operatorname{Var}(X(0), X(t)))=\operatorname{det}(\operatorname{Var}(X(0), X(t)-X(0))) .
$$

Using Lemma 3.3.

$$
p_{X(0), X(t)}(0,0) \simeq(\text { Const }) r^{-d}\left(\operatorname{det} \operatorname{Var}\left(X(0), X_{\lambda}^{\prime}(0)\right)\right)^{-\frac{1}{2}} \asymp r^{-d}
$$


where $\lambda:=t /\|t\|$. Since $S$ has been assumed to be sufficiently small, this implies that the distribution of $X(0), X(t)$ does not degenerate. We are now able to apply the Kac-Rice formula for example see [6, Theorem 6.3]. As in the case, $d=1$, we can limit our attention to the second factorial moment. We have

$$
\begin{aligned}
& \mathbb{E}((N(0, S)(N(0, S)-1)) \\
& =\int_{S^{2}} \mathcal{A}(t-s, 0) P_{X(s), X(t)}(0,0) d s d t \leq(\text { Const })|S| \int_{S} \sigma_{\max }^{2}(t)\|t\|^{-d} d t,
\end{aligned}
$$

where $|S|$ is the Lebesgue measure of $S$. Passing to polar coordinates and including $S$ in a centered ball with radius $a$, we get that the term above is bounded by

$$
\text { (Const) } \int_{0}^{a} r^{d-1} r^{-d} \sigma_{\max }^{2}(r) d r=(\text { Const }) \int_{0}^{a} \frac{\sigma_{\max }^{2}(r)}{r} d r .
$$

\subsection{General level}

In this section, we assume $\left(H_{1}\right)$ and $\left(H_{2}\right)$. Note that $\sigma_{i, \lambda}^{2}(r)$ no longer depends on $\lambda$. We denote its value by $\sigma_{i}^{2}(r)$. We have $\sigma_{\max }^{2}(r)=\max _{i=1, \ldots, d} \sigma_{i}^{2}(r)$.

Our result is the following

Theorem 3.4. Under the hypotheses above, the following are equivalent

(a) For all compact $S \subset \mathbb{R}^{d}$ and all $u \in \mathbb{R}^{d}, \mathbb{E}\left((N(u, S))^{2}\right)$ is finite.

(b) $\mathbb{E}\left((N(u, S))^{2}\right)$ is finite for some $u$ and some compact $S$ with non-empty interior. Then

(c) $\int \frac{\sigma_{\max }^{2}(r)}{r} d r$ converges at 0 .

Because of stationarity and isotropy we have

$$
\operatorname{Cov}\left(X_{i}(s), X_{i}(t)\right)=\rho_{i}\left(\|s-t\|^{2}\right),
$$

where $\rho_{i}$ is some function of class $\mathcal{C}^{2}$.

Before the proof, we state the following lemmas.

Lemma 3.5. Let $\mathcal{F}$ be a family of Gaussian distributions for $X$ and $Y$, two $d \times d$ Gaussian matrices. Let $Z$ be the $2 d^{2}$ vector obtained by the elements of $X, Y$ in any order.

(a) Suppose that for all distribution in $\mathcal{F}, \mathbb{E}(Z) \in K_{1}$ and $\operatorname{Var}(Z) \in K_{2}$, where $K_{1}$ and $K_{2}$ are two compacts sets.

Then there exists $C$ such that

$$
\sup _{f \in \mathcal{F}} \mathbb{E}_{f}(|\operatorname{det}(X) \operatorname{det}(Y)|) \leq C .
$$

The constant $C$ depends only on $K_{1}, K_{2}$ and $d$.

(b) Suppose, in addition, that for every $f \in \mathcal{F}$,

$$
\mathbb{P}\{\operatorname{det}(X)=0\}=0, \mathbb{P}\{\operatorname{det}(Y)=0\}=0,
$$

then there exists $c$ such that:

$$
\mathbb{E}(|\operatorname{det}(X) \operatorname{det}(Y)|) \geq c .
$$

The positive constant $c$ depends only on $K_{1}, K_{2}$ and $d$. 
To establish the next lemma, let us introduce the following definitions:

$$
\begin{gathered}
\sigma_{i}^{2}(r)=-2 \rho_{i}^{\prime}(0)-\frac{4 r^{2}\left(\rho_{i}^{\prime}\left(r^{2}\right)\right)^{2}}{1-\rho_{i}^{2}\left(r^{2}\right)} \\
b_{i}(r) \sigma_{i}(r)=\left(-2 \rho_{i}^{\prime}\left(r^{2}\right)-4 r^{2} \rho_{i}^{\prime \prime}\left(r^{2}\right)-\frac{4 r^{2} \rho_{i}\left(r^{2}\right)\left(\rho_{i}^{\prime}\left(r^{2}\right)\right)^{2}}{1-\rho_{i}^{2}\left(r^{2}\right)}\right) .
\end{gathered}
$$

Then we have the following, denoting by $\operatorname{Var}_{\mathcal{C}}$ the variance-covariance matrix conditional to $\mathcal{C}$.

Lemma 3.6 (See [6, p. 336]). for $r$ sufficiently small

$$
\begin{aligned}
& \operatorname{Var}_{\mathcal{C}}\left(X_{i}^{\prime}(0) ; X_{i}^{\prime}\left(r e_{1}\right)\right) \\
& \quad\left[\begin{array}{cccccccc}
\sigma_{i}(r) & 0 & \ldots & 0 & b_{i}(r) \sigma_{i}(r) & 0 & \ldots & 0 \\
0 & -2 \rho_{i}^{\prime}(0) & \ldots & 0 & 0 & 0 & \ldots & 0 \\
\ldots & \ldots & \ldots & \ldots & \ldots & \ldots & \ldots & \ldots \\
0 & 0 & \ldots & -2 \rho_{i}^{\prime}(0) & 0 & 0 & \ldots & 0 \\
b_{i}(r) \sigma_{i}(r) & 0 & \ldots & 0 & \sigma_{i}(r) & 0 & \ldots & 0 \\
0 & 0 & \ldots & 0 & 0 & -2 \rho_{i}^{\prime}(0) & \ldots & 0 \\
\ldots & \ldots & \ldots & \ldots & \ldots & \ldots & \ldots & \ldots \\
0 & 0 & \ldots & 0 & 0 & 0 & \ldots & -2 \rho_{i}^{\prime}(0)
\end{array}\right] .
\end{aligned}
$$

Proof of Theorem 3.4. Recall that $S$ can be chosen arbitrarily small so that the distribution of $X(s), X(t)$ never degenerates.

We begin by considering the quantity introduced in (3.1)

$$
\mathcal{A}(t, u)=\mathbb{E}_{\mathcal{C}}\left(\left|\operatorname{det} X^{\prime}(0) \operatorname{det} X^{\prime}(t)\right|\right) .
$$

Recall that we perform again a change of basis so that $t=r e_{1}=|t| e_{1}$ where $e_{1}$ is the first vector of the new basis. We denote the expression of $X$ in this basis by $\bar{X}$. Let $\bar{X}_{: j}^{\prime}$ denote the $j^{\text {th }}$ column of $\bar{X}^{\prime}$. Because of the independence of each coordinate assumed in $\left(H_{2}\right)$.

$$
\mathbb{E}_{\mathcal{C}}\left(\bar{X}_{i, 1}^{\prime}(0)\right)=\mathbb{E}\left(\bar{X}_{i, 1}^{\prime}(0) \mid \bar{X}_{i}(0)=\bar{X}_{i}\left(r e_{1}\right)=u_{i}\right)
$$

So, we have to consider a one-dimensional problem as in Section 2. In addition, the spectral measure of each $\bar{X}_{i}$ is invariant by isometry, so its projection on the first axis cannot be reduced to one point (or two taking into account symmetry). As a consequence, Lemma $2.3(e)$ holds implying that

$$
\mid \mathbb{E}_{\mathcal{C}}\left(\bar{X}_{i, 1}^{\prime}(0) \mid \leq(\text { Const }) u_{i} \sigma_{i}(r) .\right.
$$

Let us now consider $E_{\mathcal{C}}\left(\bar{X}_{i, j}^{\prime}(0)\right)=\mathbb{E}\left(\bar{X}_{i, j}^{\prime} \mid \bar{X}_{i}(0)=u_{i}, \frac{\bar{X}_{i}(t)-\bar{X}_{i}(0)}{r}=0\right)$ for $j \neq 1$. From Lemma 3.3

$$
\begin{aligned}
& \lim _{r \rightarrow 0} \mathbb{E}_{\mathcal{C}}\left(\bar{X}_{i, j}^{\prime}(0)\right)=\lim _{r \rightarrow 0} \mathbb{E}\left(\bar{X}_{i, j}^{\prime}(0) \mid \bar{X}_{i}(0)=u_{i}, \frac{\bar{X}_{i}(t)-\bar{X}_{i}(0)}{r}=0\right) \\
& \quad=\mathbb{E}\left(\bar{X}_{i, j}^{\prime} \mid \bar{X}_{i}(0)=u_{i}, \bar{X}_{i, 1}^{\prime}(0)=0\right) .
\end{aligned}
$$

By independence

$$
\lim _{r \rightarrow 0} \mathbb{E}_{\mathcal{C}}\left(\bar{X}_{i, j}^{\prime}(0)\right)=\mathbb{E}\left(\bar{X}_{i, j}^{\prime}(0) \mid \bar{X}_{i, 1}^{\prime}(0)=0\right)=0 .
$$

Of course, we have the same kind of result for $\bar{X}^{\prime}\left(r e_{1}\right)$. 
So, if we divide the first column of $\bar{X}^{\prime}(0)$ and $\bar{X}^{\prime}\left(r e_{1}\right)$ by $\sigma_{\max }(r)$ to obtain $\tilde{\bar{X}}^{\prime}(0)$ and $\tilde{\bar{X}}^{\prime}\left(r e_{1}\right)$, Lemma 3.6 implies that all the terms of their variance-covariance matrix are bounded and the expectation is also bounded. Using Lemma 3.5 we get that

$$
\mathcal{A}(t, u) \leq(\text { Const }) \sigma_{\text {max }}^{2}(r) .
$$

The end of the proof of the first assertion is similar to the proof of Theorem 3.1.

We turn now to the proof of the second assertion. To get the inequality in the other direction, we must carefully apply (b) of the Lemma 3.5. For this, we need to describe the compact sets, $K_{1}$ and $K_{2}$.

We know from the proof of the first assertion, that all the expectations are bounded, so $K_{1}$ is just $[-a, a]^{2 d^{2} \times 2 d^{2}}$ for some $a$. For the domain $K_{2}$ of the variance-covariance matrix of $\tilde{X}^{\prime}(0), \tilde{X}^{\prime}(t)$.

- First we have an independence between the coordinates $X_{i}$. This implies that if we denote by $X_{i::}^{\prime}(t)$ the $i^{t h}$ row of $X^{\prime}$, the gradient of $X_{i}$ at $t$, then the variables $\left(X_{i,:}^{\prime}(0), X_{i,:}^{\prime}(t)\right), i=1, \ldots, d$ are independent.

- If we consider $\left(X_{i,:}^{\prime}(0), X_{i,:}^{\prime}(t)\right)$ for some fixed $i$, we see from Lemma 3.6 that (i) only one variance varies: $\sigma_{i}(r)$, (ii) the only non-zero covariance is between $X_{i, 1}^{\prime}(0)$ and $X_{i, 1}^{\prime}(t)$.

- After dividing $X_{i, 1}^{\prime}(0)$ and $X_{i, 1}^{\prime}(t)$ by $\sigma_{\max }(r)$ to obtain $\tilde{X}_{i, 1}^{\prime}(0), \tilde{X}_{i, 1}^{\prime}(t)$, the variance becomes

$$
\tilde{\sigma}_{i}(r):=\frac{\sigma_{i}(r)}{\sigma_{\max }(r)} .
$$

The domain for these variances, when $i$ varies is

$$
K_{2}^{\prime}:=\left\{\tilde{\sigma}(r) \in \mathbb{R}^{d}: 0 \leq \tilde{\sigma}_{i}(r) \leq 1 \text {, at least one } \tilde{\sigma}_{i}(r)=1\right\} .
$$

That is a compact set.

- The domain for the covariance between $\tilde{X}_{i, 1}^{\prime}(0)$ and $\tilde{X}_{i, 1}^{\prime}(t)$ is given by CauchySchwarz inequality:

$$
\operatorname{Cov}\left(\tilde{X}_{i, 1}^{\prime}(0), \tilde{X}_{i, 1}^{\prime}(t)\right) \leq \tilde{\sigma}_{i}(r),
$$

which defines another compact set.

- The other variables being independent between them and independent of the variables above, their variances are fixed.

It remains to prove that for every element of $K_{1}$ and $K_{2}$, the Gaussian distribution satisfies

$$
\mathbb{P}\{\operatorname{det}(X)=0\}=0 \text { and } \mathbb{P}\{\operatorname{det}(Y)=0\}=0,
$$

where $X, Y$ is a representation of the conditional distribution of $\tilde{X}^{\prime}(0), \tilde{X}^{\prime}(t)$. It is sufficient to study the case of $\operatorname{det}(X)$. Recall that we have proved above that all the coordinates of $X$ are independent. The only difficulty is that the variance of the first column may vanish.

Let us consider the $d \times(d-1)$ matrix $X_{:,-1}$ that consists of columns $2, \ldots, d$ of $X$. Because all the entries of $X_{:,-1}$ are independent they span a subspace of dimension $d-1$ a.s.

Then, the rank of $X_{:,-1}$ is almost surely $(d-1)$ or in other words $\operatorname{Im}\left(X_{:,-1}\right)$ is a $(d-1)$-dimensional space. The distribution of the random matrix $Y:=X_{:,-1}$ has a density that can be written

$$
f_{Y}[d Y]=(\text { Const }) e^{-\frac{1}{2} \operatorname{Trace}\left(Y^{\top} \Sigma^{-1} Y\right)}[d Y],
$$


where $\Sigma$ is the variance-covariance matrix of each column vector, which is diagonal. This density function is translated on the Grassmannian giving a bounded density with respect to the Haar measure.

Recall that we have proved above that all the coordinates of $X$ are independent. Let $X_{:, 1}$ be the first column of $X$, conditioning on $X_{:,-1}$, by independence, the distribution of $X_{:, 1}$ remains unchanged. A representation for this random variable is

$$
X_{:, 1}=\mu+\xi, \text { with } \mu=\mathbb{E}\left(X_{:, 1}\right),
$$

where $\xi$ (because some $\tilde{\sigma}_{i}$ can vanish) has an absolute continuous distribution on the space

$$
S_{I}=\left(\xi_{i}=0, \text { for } i \in I\right) \text {, with } I=\left\{i: \tilde{\sigma}_{i}=0\right\} .
$$

But since at least one $\tilde{\sigma}_{i}=1$, we have $S_{I} \neq\{1, \ldots, d\}$.

Because of its absolute continuity, almost surely, $\xi$ cannot be included in a given subspace $E$ that does not contain $S_{I}$. In conclusion, given that it has an absolutely continuous distribution over the Grassmannian, with probability one, $\operatorname{Im}\left(X_{:,-1}\right)$ cannot contain any fixed affine space.

As a consequence, we can apply Lemma 3.5 (b) to get the inequality in the other direction.

It remains to give a lower bound to the density.

$$
p_{X(0), X(t)}(u, u)=\prod_{i=1}^{d} \frac{1}{2 \pi} \frac{1}{\sqrt{1-\rho_{i}^{2}\left(r^{2}\right)}} e^{-\frac{u^{2}}{1-\rho_{i}\left(r^{2}\right)}} .
$$

Since $\rho_{i}\left(r^{2}\right) \rightarrow 1$ as $r \rightarrow 0$, the term $e^{-\frac{u^{2}}{1-\rho_{i}\left(r^{2}\right)}}$ is lower bounded. Then it suffices to use (3.6).

\subsection{Critical points}

Let $Y$ be an isotropic random field from $\mathbb{R}^{d} \rightarrow \mathbb{R}$. Critical points of $Y$ are, in fact, zeros of $X=Y^{\prime}$. Strictly speaking, this process does not satisfy the hypotheses of Theorem 3.7 because $X^{\prime}(t)$ is the Hessian of $Y(t)$. So, it is symmetric and its distribution in $\mathbb{R}^{d^{2}}$ is not N.D. However, the result holds with a very similar proof sketched below.

Theorem 3.7. Suppose that

- $Y$ is Gaussian stationary, centered and has $\mathcal{C}^{2}$ sample paths.

- $Y^{\prime}(t)$ is N.D. The matrix $Y^{\prime \prime}(t)$ has a non degenerated distribution in the space of symmetric matrices of dimension $d \times d$.

Set

$$
\bar{S}_{\max }^{2}(r):=\max _{i=1, \ldots, d} \max _{\lambda \in \mathbb{S}^{d-1}} \operatorname{Var}\left(Y_{i \lambda}^{\prime \prime} \mid Y^{\prime}(0), Y^{\prime}(\lambda r)\right)
$$

If

$$
\int \frac{\bar{S}_{\max }^{2}(r)}{r} d r \text { converges at } 0,
$$

then for all compact $S \subset \mathbb{R}^{d}$, the second moment of the number of critical points of $Y$ included in $S$ is finite.

Remark 3.8. An analogous result was obtained in [9] under different hypothesis, but our result seems a little more general. 
Proof. (sketch) Let us define $X(t)=Y^{\prime}(t)$. Thus the matrix $X^{\prime}(t)$ is the Hessian of $Y$. This is a symmetric matrix that, we have assumed, has a non degenerated distribution. We can suppose that $X(0)=N(0, I)$. Let denote by $\mathcal{C}_{S}^{Y}$ the cardinal of the set of critical points of $Y$ included in $S \subset \mathbb{R}^{d}$. This is

$$
\#\{t \in S: X(t)=0\} .
$$

With the same notation as before let us consider the following conditional expectation

$$
\mathbb{E}_{\mathcal{C}}\left[\left|\operatorname{det}\left(X^{\prime}(0)\right) \| \operatorname{det}\left(X^{\prime}(t)\right)\right|\right]
$$

By applying the Cauchy-Schwarz inequality and by symmetry of the roles of 0 and $t$,

$$
\mathcal{A}(t, u) \leq \mathbb{E}_{\mathcal{C}}\left(\operatorname{det}\left(\left(X^{\prime}(0)\right)^{\top} X^{\prime}(0)\right)\right) .
$$

We perform a change of basis so that $t=r e_{1}=|t| e_{1}$, where $e_{1}$ is the first vector of the new basis. As $Y$ is an isotropic field, this transformation does not change its distribution. The proof continues in the same form of Theorem 3.1, substituting $\sigma_{\max }^{2}(r)$, by $\bar{S}_{\max }^{2}(r)$.

\section{Random fields from $\mathbb{R}^{D}$ to $\mathbb{R}^{d}, d<D$}

In this section, we study the level sets of a random field, $X: \mathbb{R}^{D} \rightarrow \mathbb{R}^{d}$. Of course the case, $d>D$ has no interest, because almost surely the level set is empty. The case $d=D$ has been considered in the preceding sections. So, we assume $1 \leq d<D$. The result presented here is, in some sense, a by-product of Theorem 3.4, but by its simplicity, it is the most surprising result and one of the main results of this paper.

Theorem 4.1. Let $X: X(t)$ a stationary random field $X: \mathbb{R}^{D} \rightarrow \mathbb{R}^{d}, d<D$, with $C^{(\min (2, d))}$ paths. The implicit function theorem implies that, a.s. for every $u$, the level set $C_{u}$ is a manifold, and its $(D-d)$-dimensional measure $\sigma_{D-d}\left(C_{u}\right)$, is well defined. Let $C(u, S)$ be the restriction of $C_{u}$ to a compact set $S \subset \mathbb{R}^{D}$. Assume that the distributions of $X(t)$ and $X^{\prime}(t)$ are N.D.

Then, for every $u$ and $S$,

$$
\mathbb{E}\left(\sigma_{D-d}^{2} C(u, S)\right)<+\infty .
$$

Remark 4.2. Theorem 4.1 of the article [4] contains a result for $d=1$, which is a little less general, that the one obtained using this theorem.

Proof. Using the arguments as in the proof Theorem 3.4, we prove that $X(s), X(t)$ is N.D. Kac-Rice formula reads

$$
\begin{aligned}
\mathbb{E}\left(\sigma_{D-d}^{2} C(u, S)\right) & \\
=\int_{S^{2}} \mathbb{E}_{\mathcal{C}}\left(\left(\operatorname{det}\left(X^{\prime}(s) X^{\prime}(s)^{\top}\right) \operatorname{det}\left(X^{\prime}(t) X^{\prime}(t)^{\top}\right)\right)^{\frac{1}{2}}\right) & p_{X(s), X(t)}(u, u) d s d t,
\end{aligned}
$$

where $\mathbb{E}_{\mathcal{C}}$ denotes the expectation conditional to $\mathcal{C}=\{X(0)=X(t)=u\}$. As in the proof of Theorem 3.4, we have

$$
p_{X(0), X(t)}(u, u) \leq(\text { Const })\|t\|^{-d} .
$$

By, Cauchy-Schwarz inequality and symmetry,

$$
\begin{aligned}
& A(t, u):=\mathbb{E}_{\mathcal{C}}\left(\left(\operatorname{det}\left(X^{\prime}(0) X^{\prime}(0)^{\top}\right) \operatorname{det}\left(X^{\prime}(t) X^{\prime}(t)^{\top}\right)\right)^{\frac{1}{2}}\right) \\
& \leq \mathbb{E}_{\mathcal{C}}\left(\operatorname{det}\left(X^{\prime}(0) X^{\prime}(0)^{\top}\right)\right)
\end{aligned}
$$


Using (3.2), we have to bound

$$
\mathbb{E}_{\mathcal{C}} \prod_{i=1}^{d}\left\|\nabla X_{i}(0)\right\|^{2}
$$

Now we borrow results from the proof of Theorem 3.4, to get that for every $i$ :

$$
\begin{aligned}
& \mathbb{E}_{\mathcal{C}}\left(X_{i, 1}^{\prime}(0)\right) \rightarrow 0 \\
& \mathbb{E}_{\mathcal{C}}\left(X_{i, j}^{\prime}(0)\right) \text { is bounded } j \neq 1
\end{aligned}
$$

Because of the contracting property of the conditional expectation, $\operatorname{Var}_{\mathcal{C}}\left(X_{i, j}^{\prime}(0)\right)$ is bounded. So, it follows directly that $\mathcal{A}(t, u)$ is upper-bounded. The integrability of $\|t\|^{-d}$ in $\mathbb{R}^{D}$ gives the result.

\section{A Appendix}

Proof of Lemma 2.2. Let us consider the integral

$$
\int_{0}^{\delta} \frac{\sigma^{2}(\tau)}{\tau} d \tau
$$

For $\tau$ small enough

$$
\frac{\sigma^{2}(\tau)}{\tau} \sim\left(\frac{1}{\lambda_{2}}\right) \frac{\lambda_{2}\left(1-r^{2}(\tau)\right)-\left(r^{\prime}(\tau)\right)^{2}}{\tau^{3}} .
$$

Then integrating by parts

$$
\begin{aligned}
& \int_{0}^{\delta} \frac{\lambda_{2}\left(1-r^{2}(\tau)\right)-\left(r^{\prime}(\tau)\right)^{2}}{\tau^{3}} d \tau \\
& \quad=\frac{\lambda_{2}\left(1-r^{2}(\delta)\right)-\left(r^{\prime}(\delta)\right)^{2}}{2 \delta^{2}}+\int_{0}^{\delta} \frac{r^{\prime}(\tau)}{\tau}\left(\frac{-\lambda_{2} r(\tau)-r^{\prime \prime}(\tau)}{\tau}\right) d \tau
\end{aligned}
$$

Hence, we need to consider the second term that is equal to

$$
-\lambda_{2} \int_{0}^{\delta} \frac{r^{\prime}(\tau)}{\tau}\left(\frac{r(\tau)-1}{\tau}\right) d \tau-\int_{0}^{\delta} \frac{r^{\prime}(\tau)}{\tau}\left(\frac{r^{\prime \prime}(\tau)+\lambda_{2}}{\tau}\right) d \tau
$$

The first term is evidently convergent, the above sum is convergent if and only if

$$
\int_{0}^{\delta} \frac{r^{\prime \prime}(\tau)+\lambda_{2}}{\tau} d \tau<\infty
$$

Proof of Lemma 2.3. Short, we will write $r, r^{\prime}, r^{\prime \prime}$ instead of $r(\tau), r^{\prime}(\tau), r^{\prime \prime}(\tau)$. Items $(a)-$ $(d)$ are easy consequences of regression formulas (see [6] page 100 for example).

To prove $(e)$, we study first the behavior of $\frac{r^{\prime}}{\sigma(\tau)}$ near to zero. We need, consider two cases.

The first one is when the fourth spectral moment $\lambda_{4}$ is finite: we have $r(t)=1-$ $\lambda_{2} t^{2} / 2+\lambda_{4} t^{4} /(4 !)+o\left(t^{4}\right)$. By using a Taylor expansion of fourth order on the numerator and the denominator of the fraction $\left(\frac{r^{\prime} u}{(1+r) \sigma(\tau)}\right)^{2}$, we obtain

$$
\left(\frac{r^{\prime} u}{(1+r) \sigma(\tau)}\right)^{2} \rightarrow \frac{\lambda_{2}^{2} u^{2}}{\lambda_{4}-\lambda_{2}^{2}} \leq(\text { Const }) u^{2}, \quad \text { giving (e) }
$$

Consider now the second case: $\lambda_{4}=+\infty$. 
Given that $r^{\prime \prime}(\tau)-r^{\prime \prime}(0)=2 \int_{0}^{\infty}(1-\cos (\tau \lambda)) \lambda^{2} d \mu(\lambda)$, we have by Fatou's lemma

$$
\begin{aligned}
\liminf _{\tau \rightarrow 0} \frac{\left.r^{\prime \prime}(\tau)-r^{\prime \prime}(0)\right)}{\tau^{2}} & \geq \int_{0}^{+\infty} \liminf _{\tau \rightarrow 0} \frac{1-\cos (\tau \lambda)}{\tau^{2} \lambda^{2} / 2} \lambda^{4} d \mu(\lambda) \\
& =\int_{0}^{\infty} \lambda^{4} d \mu(\lambda)=+\infty
\end{aligned}
$$

Since $1+r$ tends to 2 we get

$$
\lim _{\tau \rightarrow 0} \frac{r^{\prime 2}}{\sigma^{2}(\tau)}=\lim _{\tau \rightarrow 0} \frac{\left(1-r^{2}\right)\left(r^{\prime}\right)^{2}}{\tau^{4} \lambda_{2}^{3}} \lim _{\tau \rightarrow 0} \frac{\lambda_{2}^{3}}{\frac{\lambda_{2}\left(1-r^{2}\right)-r^{\prime 2}}{\tau^{4}}}=2 \lim _{\tau \rightarrow 0} \frac{\lambda_{2}^{3}}{\frac{\lambda_{2}\left(1-r^{2}\right)-r^{\prime 2}}{\tau^{4}}} .
$$

Note that $\lambda_{2}\left(1-r^{2}(\tau)\right)-r^{\prime 2}(\tau)=2 \lambda_{2}(1-r(\tau))-r^{\prime 2}(\tau)+O\left(\tau^{4}\right)$. Furthermore by using the l'Hospital rule

$$
\lim _{\tau \rightarrow 0} \frac{2 \lambda_{2}(1-r(\tau))-r^{\prime 2}(\tau)}{\tau^{4}}=\lim _{\tau \rightarrow 0}\left(\frac{-r^{\prime}(\tau)}{2 \tau}\right)\left(\frac{r^{\prime \prime}(\tau)-r^{\prime \prime}(0)}{\tau^{2}}\right)=+\infty,
$$

because of (A.1) and since we know that $\frac{-r^{\prime}(\tau)}{2 \tau} \rightarrow \frac{\lambda_{2}}{2}$. Thus $\frac{r^{\prime}(\tau)}{\sigma(\tau)} \rightarrow 0$. These two results imply that (e) holds.

Proof of Lemma 2.4. We can write

$$
Y_{2}-m_{2}=\rho\left(Y_{1}-m_{1}\right)+\sqrt{1-\rho^{2}} Z_{1}
$$

where $Z_{1}$ is a standard Gaussian independent of $Y_{1}$. Thus

$$
\begin{aligned}
Y_{1} Y_{2}= & \left(m_{1} m_{2}+\rho\right)+\left(m_{2}+\rho m_{1}\right)\left(Y_{1}-m_{1}\right) \\
& +m_{1} \sqrt{1-\rho^{2}} Z_{1}+\rho\left(\left(Y_{1}-m_{1}\right)^{2}-1\right)+\sqrt{1-\rho^{2}}\left(Y_{1}-m_{1}\right) Z_{1} .
\end{aligned}
$$

This formula yields that $\mathbb{E}\left|Y_{1} Y_{2}\right|$ is a continuous function of $\left(m_{1}, m_{2}, \rho\right)$ and is upperbounded by compactness.

In the other direction, $Y_{1} Y_{2}$ has an absolutely continuous density if their correlation satisfies $|\rho|<1$ and for $\rho= \pm 1$ the r.v. is equal to $\pm Y_{1}^{2}$. Then as a consequence $\mathbb{E}\left|Y_{1} Y_{2}\right|>0$ and is lower-bounded by compactnes

Proof of Lemma 3.3. Note that for $n$ large enough, the distribution of $Z_{n}$ is N.D. As a consequence,

$$
\begin{aligned}
\mathbb{E}\left(T \mid Z_{n}=z\right) & =\operatorname{Cov}\left(T, Z_{n}\right)\left(\operatorname{Var}\left(Z_{n}\right)\right)^{-1} z \\
\operatorname{Var}\left(T \mid Z_{n}\right) & =\operatorname{Var}(T)-\operatorname{Cov}\left(T, Z_{n}\right)\left(\operatorname{Var}\left(Z_{n}\right)\right)^{-1} \operatorname{Cov}\left(Z_{n}, T\right),
\end{aligned}
$$

but

$$
\operatorname{Var}\left(Z_{n}\right) \rightarrow \operatorname{Var}(Z), \text { N.D. }
$$

This implies, $\left.\left(\operatorname{Var}\left(Z_{n}\right)\right)^{-1} \rightarrow(\operatorname{Var}(Z))^{-1}\right)$. The rest is plain.

Proof of Lemma 3.5. Let $\Sigma$ be the variance-covariance matrix of $Z$, and let $\mu$ be its expectation. Both vary in a compact sets $K_{1}, K_{2}$. Let $\Sigma^{\frac{1}{2}}$ be the square root of $\Sigma$ defined in the spectral way. Using the operator norm [10], it is easy to prove that $\Sigma^{\frac{1}{2}}$ is a (uniformly) continuous function of $\Sigma$. The random vector $Z$ admits the following representation,

$$
Z=\mu+\Sigma^{\frac{1}{2}} \xi, \quad \xi \stackrel{d}{=} N\left(0, I_{2 d^{2}}\right) .
$$


The function $\operatorname{det}(X) \operatorname{det}(Y)$, as a polynomial, is a continuous function of $Z$ and by consequence $\mathbb{E}(|\operatorname{det}(X) \operatorname{det}(Y)|)$ is a continuous function of $\mu, \Sigma$. The first assertion follows by compactness.

In the other direction we have by additivity

$$
\mathbb{P}\{\operatorname{det}(Y) \operatorname{det}(X)=0\}=0 .
$$

This implies that

$$
\mathbb{E}(|\operatorname{det}(X) \operatorname{det}(Y)|)>0
$$

Again, the second inequality is obtained by compactness.

\section{References}

[1] R. Adler. The geometry of random fields. John Wiley \& Sons, Ltd., Chichester (1981). MR0611857

[2] R. Adler \& A. M. Hasofer. Level crossings for random fields. Ann. Probab. 4, no. 1, 1-12 (1976). MR-0405559

[3] R. Adler \& J. Taylor. Random fields and geometry. Springer Monographs in Mathematics. Springer, New York (2007). MR-2319516

[4] D. Armentano, J.-M. Azaïs, D. Ginsbourger \& J. R. León. Conditions for the finiteness of the moments of the volume of level sets. Electron. Commun. Probab. 24, no. 17, 1-8 (2019). MR-3933041

[5] A. Auffinger \& G. Ben Arous. Complexity of random smooth functions on the high-dimensional sphere. Ann. Probab. 41, no. 6, 4214-4247 (2013). MR-3161473

[6] J.-M. Azaïs \& M. Wschebor. Level sets and extrema of random processes and fields. John Wiley \& Sons, Inc., Hoboken, NJ (2009). MR-2478201

[7] M. V. Berry. Statistics of nodal lines and points in chaotic quantum billiards: perimeter corrections, fluctuations, curvature. Journal of Physics A 35, 3025-3038 (2002). MR-1913853

[8] H. Cramer \& M. R. Leadbetter. Stationary and related stochastic processes. Sample function properties and their applications. John Wiley \& Sons, Inc. (1967). MR-0217860

[9] A. Estrade \& J. Fournier. Number of critical points of a Gaussian random field: condition for a finite variance. Statist. Probab. Lett. 118, 94-99 (2016). MR-3531487

[10] Yu. B. Farforovskaya \& L. Nikolskaya. Modulus of continuity of operator functions. St. Petersburg Math. J. 20, no. 3, 493-506 (2009). MR-2454458

[11] D. Geman. On the variance of the number of zeros of a stationary Gaussian process. Ann. Math. Statist. 43, 977-982 (1972). MR-0301791

[12] M. Kac. On the average number of real roots of a random algebraic equation. Bull. Amer. Math. Soc. 49, 282-332 (1944). MR-0007812

[13] M. Kratz \& J. R. León. On the second moment of the number of crossings by a stationary Gaussian process. Ann. Probab. 34, no. 4, 1601-1607 (2006). MR-2257657

[14] M. Kratz \& J. R. León. Central limit theorems for level functionals of stationary Gaussian processes and fields. Journal of Theoretical Probability 14, no. 3 639-672 (2001). MR-1860517

[15] T. Letendre. Expected volume and Euler characteristic of random submanifolds. J. Funct. Anal. 270, no. 8, 3047-3110 (2016). MR-3470435

[16] G. Lindgren \& F. Lindgren. Stochastic asymmetry properties of 3D Gauss-Lagrange ocean waves with directional spreading. Stochastic Models 27, 490-520 (2011). MR-2827442

[17] D. Marinucci, G. Peccati, M. Rossi \& I. Wigman. Non-universality of nodal length distribution for arithmetic random waves. Geom. Funct. Anal. 26, no. 3, 926-960 (2016). MR-3540457

[18] S. O. Rice. Mathematical analysis of random noise I. Bell System Tech. J. 23, 314-320 (1943). MR-0010932 
On the second moment of the measure of level sets

[19] S. O. Rice. Mathematical analysis of random noise II. Bell System Tech. J. 24, 46-156 (1945). MR-0011918

[20] M. Wschebor. Mésure géométrique des ensembles de niveau. LNM, vol. 1147. Springer-Verlag (1985). MR-0871689

Acknowledgments. The authors thank an anonymous referee for careful reading and precise observations that made this article improve considerably. 


\section{Electronic Journal of Probability Electronic Communications in Probability}

\section{Advantages of publishing in EJP-ECP}

- Very high standards

- Free for authors, free for readers

- Quick publication (no backlog)

- Secure publication $\left(\mathrm{LOCKSS}^{1}\right)$

- Easy interface (EJMS²)

\section{Economical model of EJP-ECP}

- Non profit, sponsored by $\mathrm{IMS}^{3}, \mathrm{BS}^{4}$, ProjectEuclid ${ }^{5}$

- Purely electronic

\section{Help keep the journal free and vigorous}

- Donate to the IMS open access fund ${ }^{6}$ (click here to donate!)

- Submit your best articles to EJP-ECP

- Choose EJP-ECP over for-profit journals

\footnotetext{
${ }^{1}$ LOCKSS: Lots of Copies Keep Stuff Safe http://www. lockss.org/

${ }^{2}$ EJMS: Electronic Journal Management System http://www.vtex.lt/en/ejms.html

${ }^{3}$ IMS: Institute of Mathematical Statistics http://www.imstat.org/

${ }^{4}$ BS: Bernoulli Society http://www. bernoulli-society.org/

${ }^{5}$ Project Euclid: https://projecteuclid.org/

${ }^{6}$ IMS Open Access Fund: http://www.imstat.org/publications/open.htm
} 